\title{
Itinéraires alexandrins
}

\section{Christian Jacob}

\section{(2) OpenEdition \\ Journals}

Édition électronique

URL : https://journals.openedition.org/rbnu/3339

DOI : $10.4000 /$ rbnu.3339

ISSN : 2679-6104

\section{Éditeur}

Bibliothèque nationale et universitaire de Strasbourg

\section{Édition imprimée}

Date de publication : 1 novembre 2010

Pagination : 64-69

ISSN : 2109-2761

\section{Référence électronique}

Christian Jacob, «Itinéraires alexandrins », La Revue de la BNU [En ligne], 2 | 2010, mis en ligne le 01 novembre 2010, consulté le 08 août 2021. URL : http://journals.openedition.org/rbnu/3339 ; DOI : https://doi.org/10.4000/rbnu.3339

\section{(c) (i) (3)(2)}

La Revue de la BNU est mise à disposition selon les termes de la Licence Creative Commons Attribution - Pas d'Utilisation Commerciale - Partage dans les Mêmes Conditions 4.0 International. 


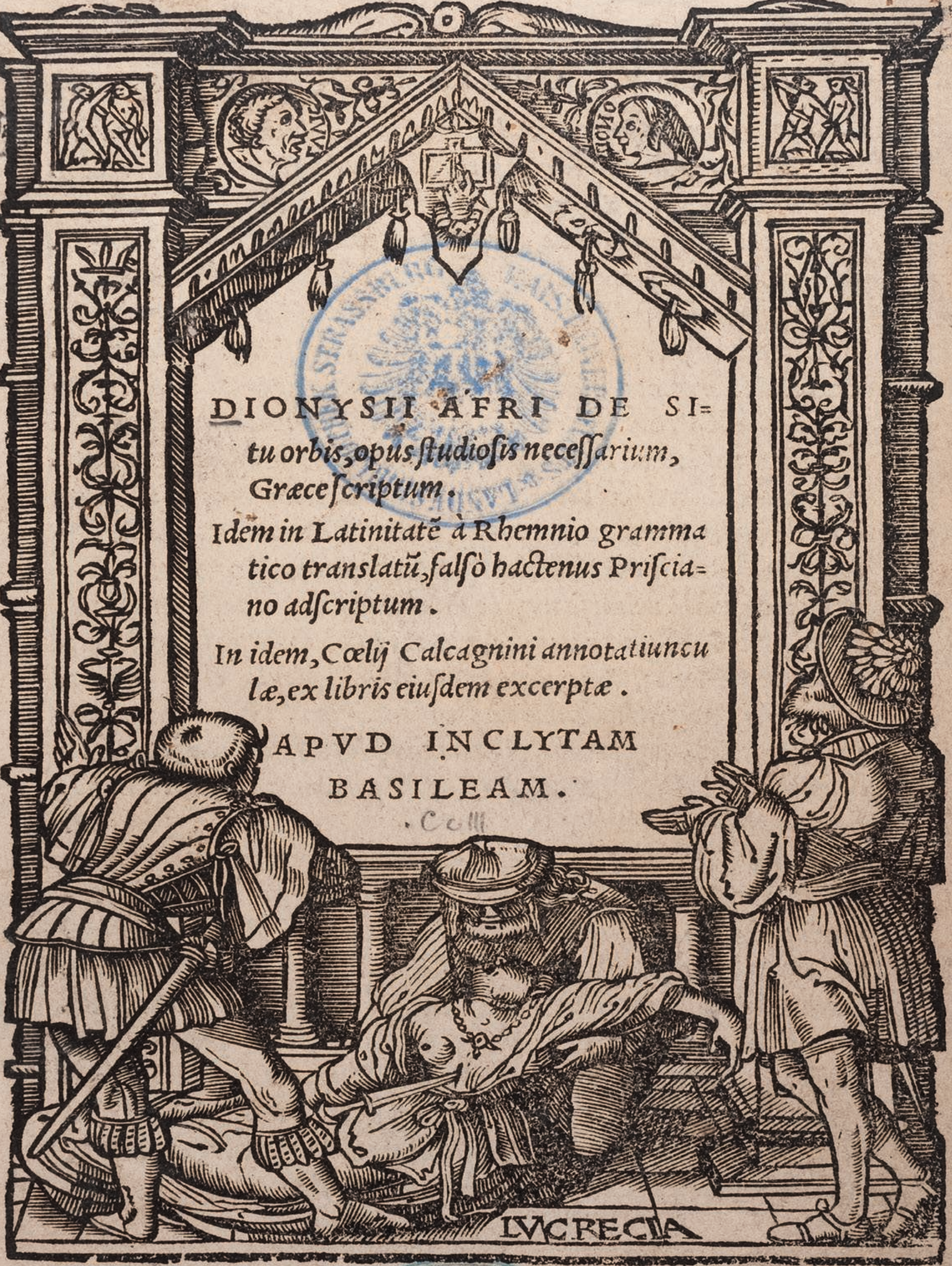




\section{Itinéraires alexandrins}

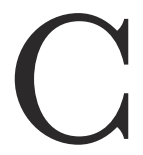

omment mon parcours d'historien m'at-il conduit à devenir un alexandrin et à faire de la mythique bibliothèque des Ptolémées l'un de mes lieux d'ancrage, entre savoir et imaginaire?

A la fin des années 70 du siècle dernier, je m'apprêtais à suivre des chemins différents. Je rêvais de devenir archéologue, j'aspirais à préparer l'Ecole française d'Athènes. J'étais fasciné par le monde des objets et des formes, par les pierres et la terre, par les céramiques et le marbre. Les sites archéologiques me semblaient être comme des livres dont je serais le premier lecteur, découvrant page après page les signes et les traces abandonnés et superposés il y a plus de deux millénaires.

Ce livre inscrit dans le sol m'apparaissait comme l'un de ces manuscrits médiévaux où l'on réemploie le support précieux du parchemin en effaçant un texte pour en inscrire un autre. Le sol était un palimpseste, sur lequel, de la préhistoire à nos jours, avaient été inscrits les travaux et les jours, le quotidien et l'histoire, la routine et l'événement, à travers des traces volontaires ou accidentelles.

Je rêvais l'archéologie comme un art particulier de la lecture, les sites me semblaient être des bibliothèques : Delphes et Délos, l'agora d'Athènes et Olympie... Le carré de fouilles, délimité selon les règles de l'art, était comme la page d'un livre de cette bibliothèque, qui me raconterait une histoire singulière si j'apprenais les règles de son déchiffrement. Chaque bibliothèque, chaque livre avait son propre langage, sa propre histoire...

Je me suis même orienté pour un temps vers les formes premières de ces littératures inscrites dans le sol grec : Mycènes, Santorin, Cnossos et Phaïstos. Sans doute parce que les disciplines du déchiffrement étaient plus exigeantes, plus ascétiques, il fallait " lire " des textes inscrits non sur les rouleaux de papyrus ou les codices de parchemin, mais sur des tablettes d'argile, non pas dans l'alphabet grec, mais dans des répertoires de signes syllabaires que l'on distinguait en " linéaire A » et " linéaire B ».

L'histoire du déchiffrement du linéaire B par Michael Ventris et John Chadwick m'avait passionné ${ }^{1}$. Et j'avais mis à apprendre ces signes syllabiques la même passion que l'on aurait pu mettre face aux écritures de la Mésopotamie ou de la Chine : j'étais au seuil d'une bibliothèque à conquérir... Certes, les textes offerts à la lecture étaient le plus souvent des inventaires, des listes, mais quelle émotion de voir émerger une forme de langue grecque primitive au fur et à mesure du déchiffrement : " Ai-ke-u ke-resio ti-ri-po-de we-ke ", "Egée le Crétois a fabriqué un trépied "...

Alors que je me consacrais à l'apprentissage de cette écriture et que je tentais à mon tour de briser le code des signes du " disque de Phaïstos ", connus par un seul artefact, vint la rencontre avec d'autres grands lecteurs. Ils avaient pour noms Jean-Pierre Vernant, Marcel Detienne et Pierre Vidal-Naquet Nicole Loraux vint se joindre à eux très vite. Je les avais déjà lus, il me fut donné de les entendre et d'assister à leurs séminaires. Lieux étranges de déchiffrement, de lecture et d'interprétation, où les textes classiques que j'avais travaillés pendant mes années d'études, avec l'énergie, mais aussi l'étroitesse de vue d'un laboureur ou d'un bûcheron, prenaient vie et couleurs, sens et relief, se mettaient à parler et à penser... Ils ouvraient des horizons, invitaient à tracer des cheminements interprétatifs, composaient des paysages signifiants aux multiples harmoniques... 
Le monde que je découvris alors était aussi dépaysant que le rivage d'un nouveau continent, il était plein de couleurs et de sons, de paradoxes et d'énigmes. Les Grecs de Detienne et Vernant ne ressemblaient pas à ceux que j'avais croisés dans mon itinéraire universitaire, ils vivaient et pensaient, ils chantaient et imaginaient, et les textes comme les images devenaient des partitions à déchiffrer...

Le livre de Marcel Detienne, Les Jardins d'Adonis. La mythologie des aromates en Grèce ancienne ${ }^{2}$, puis celui qu'il écrivit en collaboration avec Jean-Pierre Vernant, Les ruses de l'intelligence, la Mètis des Grecs ${ }^{3}$ m'ouvrirent les portes d'une bibliothèque insoupçonnée : à côté des orateurs attiques et de Platon, d'Homère et des tragiques, apparaissait une foule d'auteurs inconnus, des érudits, des lexicographes, des scholiastes, des compilateurs et des encyclopédistes. Sous forme de fragments ou de citations, ou parfois de traités techniques et scientifiques, ces auteurs dévoilaient

J'étais l'un de ceux qui furent intéressés davantage par l'estuaire que par le cours supérieur des fleuves de l'érudition antique méritaient davantage qu'un statut ancillaire dans cette anthropologie des mondes antiques en émergence. Qui étaient ces érudits ? Comment travaillaient-t-ils ? Comment articulaient-ils les étapes de lecture et de rédaction dans leur travail ? Comment procédaient-ils concrètement, quelle était l'ergonomie du travail savant au temps des rouleaux de papyrus et des tablettes de cire ?

Ces questions très générales, je les formulais une première fois dans un mémoire consacré à la Périégèse de la Grèce de Pausanias, une description des cités grecques et de leurs monuments à l'époque antonine. J'explorais la construction et la syntaxe du texte, dont une lecture approfondie m’avait révélé la cohérence. Je percevais, au-delà de la poussière des routes grecques et des notes prises in situ, le rôle des bibliothèques fréquentées par Pausanias. En prêtant une oreille attentive, je saisissais même la musique particulière de son usage des sources, écrites ou orales, qui tantôt pouvaient résonner à l'unisson (" sumphôneîn ") ou créer des discordances (" diaphôneîn "). Je

fus fasciné par cette métaphore sonore. Les livres des bibliothèques ont leur musique propre. Ils peuvent s'harmoniser ou créer de multiples dissonances. La "symphonie " et la "diaphonie " des sources et des savoirs ouvrent un espace d'écoute, de comparaison, de critique, de décision.

Je poursuivis mon cheminement par une thèse de doctorat consacrée à un géographe grec mineur, Denys d'Alexandrie, auteur d'une Périégèse de la Terre habitée et contemporain de l'empereur Hadrien. Je me retrouvais à Alexandrie à l'époque impériale : la ville avait perdu un peu de ses fastes et de son rayonnement, mais elle restait un pôle de culture et de savoirs, une ville-mémoire où se croisaient de multiples traditions.

Le texte de Denys est un poème didactique écrit dans un grec littéraire où se mêlent les usages homériques et les formes sophistiquées des grands poètes alexandrins. Il offrait aux élèves des écoles 
une description de l'ensemble du monde connu, en entrelaçant aux listes de toponymes et de noms de peuples des données mythographiques, ethnographiques et religieuses. J'eus très vite la conviction que son propos était d'offrir une version condensée de la bibliothèque géographique antérieure, dont les savoirs se trouvaient résumés et traduits dans un cadre cohérent et mémorisable. De la bibliothèque au texte, du texte à la bibliothèque : le poème de Denys parvenait à dérouler des généalogies signifiantes, à déployer des réseaux de références et d'allusions qui le reliaient aux grandes œuvres de la culture grecque, comme les épopées homériques, Hésiode, Callimaque ou les Argonautiques d'Apollonios de Rhodes. Chaque vers, chaque mot semblait porteur d'une mémoire, qui le reliait à d'autres textes, qui le chargeait de sens et d'intentionnalité. La brièveté de cette description du monde contrastait avec l'expansion herméneutique à laquelle elle invitait.

Tous ces fils me conduisaient à l'âge d'or alexandrin, au III ${ }^{e}$ siècle avant notre ère, au Musée ${ }^{5}$ et à sa collection de livres. Je rencontrais d'abord Eratosthène de Cyrène : polymathe et philosophe, il prit la tête de la bibliothèque et mena dans ce cadre un vaste ensemble de travaux mathématiques, astronomiques, grammaticaux et géographiques. C'est l'usage critique des ressources de la bibliothèque qui lui permit de " corriger " les anciennes cartes de la terre, comme d'autres corrigeaient le texte d'Homère. Sa Géographie, connue seulement par la tradition indirecte, mobilisait une documentation nouvelle, notamment sur l'Asie, recueillie lors de l'expédition d'Alexandre. Les données littéraires, les cartes antérieures, les mesures des itinéraires et des périples étaient soumises à une critique aussi rigoureuse que possible, puis traduites en formes schématiques, en alignements, en écarts sur une carte régie par la géométrie euclidienne. Les données partielles, hétérogènes et empiriques fournies par les voyageurs et les explorateurs étaient transformées et épurées, réunies dans une synthèse graphique, dans une "vue de l'esprit " par le cartographebibliothécaire.

Autre cartographe, Callimaque de Cyrène, qui sans avoir le titre de "bibliothécaire " eut la charge de superviser une forme de catalogue des collections royales, les Tables de tous ceux qui se sont illustrés dans le domaine de la culture et de ce qu'ils ont écrit, en 120 rouleaux. Poète virtuose qui trouvait son inspiration dans ses innombrables lectures, dans les variantes des anciens mythes comme dans les mille curiosités lexicales que l'on pouvait glaner chez les auteurs d'autrefois, il fut aussi l'auteur d'une œuvre érudite qui prenait volontiers la forme de listes et de catalogues regroupant ses trouvailles de lecteur par grands champs thématiques, qu'il s'agisse de proverbes, de mirabilia, de mots rares et dialectaux que l'on employait dans les cités grecques. Cette érudition de grand lecteur reproduisait dans le texte érudit la logique cumulative de la bibliothèque. Cette dernière apparaissait comme un vaste gisement de connaissances, de mots et de textes, un horizon à explorer et à mettre en ordre, pour faire surgir, de l'accumulation des livres, un espace organisé selon les découpages des savoirs et des genres littéraires. On ne peut travailler sur la culture hellénistique sans rêver à la bibliothèque des Ptolémées, sans devenir soi-même un alexandrin. De la bibliothèque elle-même, il ne reste aucun vestige matériel, nous en sommes réduits à des conjectures et à des comparaisons pour esquisser un cadre architectural et des dispositifs de rangement. Nous n'avons que de très rares données sur son organisation, son personnel, ses usages. Si la liste de ses "directeurs" nous est connue, nous n'avons aucune information sur les scribes qui recopiaient les textes, sur les modalités d'accès aux livres, sur les lieux où travaillaient les lecteurs. Seule certitude : la bibliothèque d'Alexandrie ne comportait pas de salle de lecture, que l'on trouve dès les bibliothèques publiques de la Rome impériale. Elle était au sens propre l'espace de rangement et de conservation des rouleaux de papyrus. Ses usagers appartenaient à l'entourage royal, au cercle savant et lettré du Musée. Ce n'était donc pas une "bibliothèque publique " à la disposition de tous. Il ne faut pas imaginer les lecteurs absorbés dans leurs livres, assis devant des tables. La gestuelle, la mobilité, l'environnement sonore (on lisait à voix haute ou murmurée), les discussions entre lecteurs savants, tout cela échappe aux sources qui nous sont parvenues.

Dès l'Antiquité, la bibliothèque d'Alexandrie a donné lieu à une tradition légendaire, qu'il s'agisse de sa fondation, liée à la traduction en grec de la Torah, ou de l'incendie qui aurait détruit ses collections, imputé à Jules César. Les récits sont repris d'un auteur à l'autre, passent du grec au latin, du 


\section{On ne peut travailler sur la culture hellénistique sans rêver à la bibliothèque des Ptolémées, sans devenir soi-même un alexandrin}

grec à l'arabe, entre païens, juifs, chrétiens et musulmans, ils se métamorphosent dans un jeu complexe d'échos, de contresens involontaires, d'arrière-pensées apologétiques. La tradition est labyrinthique, et l'historien doit démêler les fils, reconstituer les sources et les relais de ces récits, retrouver le noyau factuel à l'origine des variantes mythiques. Tel a été le cheminement suivi par Luciano Canfora, dans deux ouvrages majeurs consacrés à la tradition de la bibliothèque ${ }^{6}$.

Une autre approche, non moins fascinante, conduit à observer l'impact de la bibliothèque d'Alexandrie sur la culture hellénistique et romaine. On observe alors les ondes de choc, superficielles ou profondes, politiques et institutionnelles ou intellectuelles et littéraires, qui se propagent dans le temps et dans l'espace depuis l'épicentre alexandrin. Le rôle de la fondation des Ptolémées apparaît pour ainsi dire en creux, dans la création de bibliothèques concurrentes dans d'autres capitales hellénistiques puis à Rome, dans le développement des bibliothèques privées et d'un intérêt bibliophilique pour les livres rares et anciens, dans la constitution d'instruments bibliographiques permettant d'inventorier les textes et de déjouer les pièges d'auteurs anonymes, dans la diffusion de formes particulières d'érudition grammaticale et antiquaire, dans le développement de la philologie qui travaille à reconstruire les textes à partir de leurs variantes. Le travail intellectuel, les pratiques lettrées et savantes ne sont bien sûr pas nés à Alexandrie, ni même en Grèce. Mais la bibliothèque d'Alexandrie, en réunissant le patrimoine écrit de l'hellénisme et les grands textes représentatifs des " sagesses barbares ", et en offrant au cercle des membres du Musée une collection de livres d'une ampleur sans précédent, marque un seuil dans l'histoire des savoirs et des sciences. La bibliothèque apporte un immense gisement de textes et de connaissances, elle est elle-même enrichie par toute la littérature savante qu'elle permet de produire : commentaires, compilations, traités scientifiques, lexiques, éditions critiques, monographies sur les sujets les plus divers. Les érudits postérieurs, qu'il s'agisse de Pline l'Ancien ou d'Athénée de Naucratis, de Plutarque, du médecin Galien ou du géographe Strabon, pour me limiter à quelques noms, témoignent de ces pratiques lettrées, où la lecture est indissociable de l'écriture, où les livres sont des champs de connaissances à moissonner, des gisements de problèmes à identifier et à résoudre, des sources qui permettent de remonter dans le temps et de voyager dans l'espace. Lire, prendre des notes, réorganiser ces notes dans un nouveau texte, citer et critiquer, expliquer, mémoriser, commenter, sont autant d'opérations constitutives de leur travail savant. Le travail sur les textes et sur les livres, cependant, ne représentait qu'un versant de leurs activités : le dialogue, les propos de table, les cours et conférences, les controverses parfois, dessinent le champ de leurs sociabilités savantes, d'une communauté de savoirs et d'intérêts où se décèlent encore la musique propre des mondes lettrés, la symphonie et la diaphonie des textes, de leurs auteurs et de leurs lecteurs.

Telles sont les perspectives de recherche que j'ai choisi de suivre ces dernières années. J'ai tenté de comprendre, dans leur spécificité et leur altérité, les formes de travail des lettrés de l'époque hellénistique et impériale, en remontant aussi loin que possible des textes vers les opérations qui les avaient produits, des livres vers les pratiques de lecture qu'ils présupposaient. Le milieu de bibliophiles érudits mis en scène par Athénée de Naucratis à Rome, à la fin du II ${ }^{e}$ siècle de notre ère, m'a offert un terrain d'observation aussi dépaysant qu'une tribu de la forêt amazonienne ${ }^{7}$. Mais ce tropisme bien alexandrin de l'érudition historique et philologique m'a aussi conduit à formuler des questionnements plus généraux et fondamentaux, sur la nature, la finalité, les pratiques du travail savant, sur le 
va-et-vient entre la main et l'intelligence, entre les instruments et la pensée, entre les textes et la mémoire, questionnements que j’ai déployés dans un projet interdisciplinaire et comparatiste, les Lieux de savoir ${ }^{8}$.

La bibliothèque d'Alexandrie reste pour moi une énigme historique qu'il faut tenter d'élucider par une patiente archéologie des témoignages écrits, à défaut de pouvoir retrouver ses fondations dans le sol. Elle est aussi le paradigme d'une certaine forme de travail savant, qui a irrigué les traditions de l'Occident et de l'Orient : un modèle où la lecture et l'écriture sont indissociables, où les savoirs se construisent, se matérialisent et se transmettent dans ces supports matériels et efficaces que sont les livres.

Le mythe et l'histoire de la bibliothèque d'Alexandrie, enfin, peuvent éclairer les grandes mutations techniques, politiques et sociales qui reconfigurent aujourd'hui le champ des savoirs, de l'écrit, de l'information et de la mémoire. L'une de ces leçons, très actuelle, est que l'accumulation indéfinie, l'aspiration à l'universalité, le fantasme de la totalité ne sont que de vaines illusions et conduisent à l'amnésie et au naufrage de la pensée, sans l'ensemble des pratiques qui réintroduisent du sens, de l'ordre et de la visibilité, qui organisent l'espace et le temps et mettent en perspective les gisements de l'écrit, du son et de l'image.

Comme dans la métropole des Ptolémées, les conservateurs de bibliothèque ont un rôle essentiel à jouer, au cœur des communautés savantes d'aujourd'hui et de demain.

\section{Christian Jacob}

\section{Notes :}

1 - Voir John Chadwick, Le déchiffrement du linéaire B. Aux origines de la langue grecque, Paris, Gallimard, 1972

2 - Paris, Gallimard, 1972

3 - Paris, Flammarion, 1974

4 - Le Centre de recherches comparées sur les sociétés anciennes, devenu Centre Louis Gernet, et aujourd'hui Anhima (Anthropologie et histoire des mondes antiques).

5- Le Musée était un centre de recherches littéraires et scientifiques fondé par les Ptolémées à Alexandrie. Hébergé dans leur palais royal, c'est en son sein que fut créée la fameuse bibliothèque.

6 - La véritable histoire de la bibliothèque d'Alexandrie, Paris, Desjonquères, 1988 ; Il viaggio di Aristea, Roma-Bari, Laterza, 1996

7 - "Ateneo, o il Dedalo delle parole", in : Ateneo, I Deipnosofisti. I dotti a banchetto, Roma, Salerno Editrice, 2001, vol. 1, pp. XI-CXVI

8 - Lieux de savoir, I. Espaces et communautés, Paris, Albin Michel, 2007 ; II. Les mains de l'intellect, Paris, Albin Michel, à paraître en janvier 2011 